\title{
Sankofa pediatric HIV disclosure intervention did not worsen depression scores in children living with HIV and their caregivers in Ghana
}

Christopher Radcliffe ${ }^{1}$, Aba Sam¹, Quinn Matos', Sampson Antwi², Kofi Amissah², Amina Alhassan², Irene Pokuaa Ofori ${ }^{2}$, Yunshan $\mathrm{Xu}^{3}$, Yanhong Deng ${ }^{3}$, Nancy R. Reynolds ${ }^{4}$, Elijah Paintsil ${ }^{* *}$ (D) and on Behalf of the Sankofa Team

\begin{abstract}
Background: The 'Sankofa' pediatric HIV disclosure study (2013-2017) was an intervention that aimed to address the low prevalence of disclosure of HIV status in Ghana.

Methods: We conducted a cross-sectional study at the intervention site in Kumasi, Ghana, in 2019, (2 years after study closure) and administered the 21-item Beck Depression Inventory (BDI) and the 10-item Child Depression Inventory (CDI) to caregiver-child dyads who received the intervention.
\end{abstract}

Results: We enrolled 65\% ( $N=157)$ of the original dyads in the present study. Between Sankofa enrollment baseline and the present study, both children and caregivers had significant $(p<0.0001)$ mean reductions in CDI scores and BDI scores, respectively. CDI scores of the children were significantly correlated with $\mathrm{BDI}$ scores of the caregivers $(r=0.019, p=0.019)$. No statistically significant associations between disclosure status and either CDI score or BDI score were found.

Conclusions: Our findings did not support caregivers' fears that disclosure leads to depression.

Trial registration: ClinicalTrials.gov Identifier: NCT01701635 (date of registration Oct 5, 2012).

Keywords: Pediatric HIV, Disclosure intervention, Depression, Ghana

\section{Background}

In 2018, there were 2.8 million children and adolescents living with HIV globally [1]. Unfortunately, sub-Saharan Africa has been disproportionately affected by the HIV epidemic, and it is home to nearly $90 \%$ of children and adolescents living with HIV [1]. There has been an unprecedented effort to scale up antiretroviral therapy (ART) in sub-Saharan Africa. With expanding ART coverage in subSaharan Africa, children living with HIV are living longer

* Correspondence: elijah.paintsil@yale.edu

'Department of Pediatrics, Pharmacology \& Public Health, Yale School of Medicine, 464 Congress Avenue, New Haven, CT 06520, USA

Full list of author information is available at the end of the article and surviving into adulthood due to declines in AIDSrelated deaths $[2,3]$. However, these gains can be reversed when adherence to ART is sub-optimal. Sustained viral suppression is the goal of optimal adherence ( $\geq 95 \%)$, and there are several efforts and strategies focused on ensuring this level of adherence along the HIV care continuum, particularly in children living with HIV $[4,5]$. Nevertheless, there are reports of poor adherence among children living with HIV leading to virologic or treatment failure $[6,7]$. Non-disclosure of HIV status to children living with HIV is a critical barrier to optimal adherence to therapy [8]. There is a growing body of evidence to support the benefits of

(c) The Author(s). 2020 Open Access This article is licensed under a Creative Commons Attribution 4.0 International License, which permits use, sharing, adaptation, distribution and reproduction in any medium or format, as long as you give appropriate credit to the original author(s) and the source, provide a link to the Creative Commons licence, and indicate if changes were made. The images or other third party material in this article are included in the article's Creative Commons licence, unless indicated otherwise in a credit line to the material. If material is not included in the article's Creative Commons licence and your intended use is not permitted by statutory regulation or exceeds the permitted use, you will need to obtain permission directly from the copyright holder. To view a copy of this licence, visit http://creativecommons.org/licenses/by/4.0/ The Creative Commons Public Domain Dedication waiver (http://creativecommons.org/publicdomain/zero/1.0/) applies to the data made available in this article, unless otherwise stated in a credit line to the data. 
disclosure, such as retention in care, avoidance of treatment failure, and improvement of HIV knowledge [7, 9, 10].

Disclosure is the process by which a child is incrementally made aware of his or her HIV status in an ageappropriate manner [11]. Despite the WHO's 2011 recommendations and guidelines that encourage disclosure, the prevalence of disclosure of HIV status to children living with HIV in sub-Saharan Africa is generally low, ranging from 9 to $72 \%$ in a recent review [11, 12]. A rate limiting factor for disclosure has been caregivers' concerns that disclosure could have negative psychological and cognitive effects in children living with HIV [13, 14]. Other barriers center on caregivers' fears that children living with HIV will tell others of their diagnoses, leading to stigmatization and isolation $[13,15,16]$. Moreover, there are no consensuses on who ought to disclose (caregiver vs. provider) and how to disclose. Few studies in sub-Saharan Africa have explored how best to disclose [14, 17, 18].

The 'Sankofa' pediatric HIV disclosure intervention was one such study that sought to address the low prevalence of disclosure in Ghana and the lack of disclosure skills among their caregivers [14, 15]. The site-randomized intervention lasted from January 2013 to June 2017, with a control site in Accra, Ghana, and an intervention site in Kumasi, Ghana. The intervention featured a disclosure model that incorporated bioecological systems theory and core elements of the Information-Motivation-Behavioral Skills model of Health Behavior Change [19-23]. The patient-centered intervention was delivered by adherence and disclosure specialists who were familiar with the community and trained to address the information, motivation, and behavioral skills of caregivers in an attempt to facilitate their engagement in the disclosure process from initiation to completion. The intervention was delivered in a sensitive fashion that considered the personal needs and cognitive capacities of children. Children in the intervention group were found to be 3.98- and 4.21-times more likely to have been disclosed to than children in the control group at one and 3 years, respectively [24].

We report the findings of a cross-sectional, postintervention study conducted from June to August 2019 that sought to explore the long-term effects of the 'Sankofa' parent study and disclosure on depression symptoms in caregiver-child dyads at the intervention site, Kumasi, Ghana. We aimed to: 1) determine any changes in depression symptoms in dyads between Sankofa baseline and the current study and 2) explore the effect of disclosure status on depression symptoms over time.

\section{Methods}

\section{Study participants}

The recruitment and organization of the parent study, 'Sankofa,' has been previously described [14]. Study enrollment for the 'Sankofa' study lasted from January
2013 till June 2016, and the study ended in 2017. It had a control arm at Korle Bu Teaching Hospital in Accra, Ghana, and an intervention arm at Komfo Anokye Teaching Hospital (KATH) in Kumasi, Ghana. All caregiverchild dyads who received the intervention at KATH were eligible for inclusion in this cross-sectional study which enrolled participants from June to August 2019. Dyads were recruited at KATH's pediatric HIV clinic during routine clinic appointments. Written informed consent was obtained from the caregivers of the children living with HIV, and consent was obtained from caregivers on behalf of children less than 18 years of age with an assent from the child participant. The study protocol was reviewed and approved by the Institutional Review Boards of Kwame Nkrumah University of Science Technology/ KATH (Protocol \#: CHRPE/AP/146/12) and Yale University (Protocol \#: 1205010310).

\section{Procedures}

The 10-item Child Depression Inventory (CDI) and the 21item Beck Depression Inventory (BDI) were administered to caregiver-child dyads in the parent Sankofa study every 48 weeks till the end of the study [25]. Other instruments such as Brief Illness Perceptions Questionnaire (Brief IPQ), Social Provisions Scale, brief HIV Knowledge questionnaire (HIV-KQ-18), and HIV Stigma Scale were administered to caregivers during the same timepoints in the parent study [26-29]. In the current study, we administered only the $\mathrm{CDI}$ and BDI to the children and caregivers, respectively. To avoid accidental disclosure, questionnaires were administered to children in the presence of their caregivers whereas caregivers were interviewed alone. The interviews took place in a clinic setting, and the majority were completed in English. For participants who only spoke Twi, the major regional language, native-speaking research staff translated accordingly. The original research staff from the Sankofa study administered the questionnaires with the help of authors AS, QM, and CR.

\section{Measures}

Demographic data for the caregiver-child dyads were collected upon enrollment in the Sankofa study. The 10-item CDI was used to screen for depression symptoms in children. Each of the 10 items is scored on a four-point scale $(0-3)$ to yield a maximum score of 30 . This abridged form of the CDI has yet to be validated in our population; however, a version of the 10-item CDI has been studied in African adolescents without HIV [30]. Cutoff scores are not available, and a higher CDI score indicates greater severity of depression symptoms. The 21-item BDI was used to assess depression symptoms in caregivers. The BDI has been previously validated in sub-Saharan Africa [31], and it rates each of its 21 items on a four-point scale $(0-3)$. A total score is assigned, and its results are divided into four 
categories depending on the severity of depression symptoms: minimal (0-9); mild (10-18); moderate (19-29); and severe (30-63). For our population, the BDI has been reported to have a three-factor structure (affective, cognitive, and somatic) and high internal reliability (0.90) [31].

\section{Analysis}

Baseline demographic data were summarized with descriptive statistics. Means and standard deviations were determined for continuous variables whereas counts and percentages were determined for categorical variables. Differences in demographic data between disclosed and nondisclosed groups were assessed using t-test or Wilcoxon rank sum test for continuous variables and chi-square or Fisher's exact tests for categorical variables, as appropriate. The same methods were used to assess differences between dyads who received the intervention and were enrolled in the current study versus those who were not enrolled. The mean changes of CDI and BDI scores between baseline and the current study were examined using paired $t$-tests. The correlation of CDI and BDI scores was examined using Pearson's correlation coefficients. The changes in BDI categories, defined by standard cutoff scores, were also evaluated using McNemar's test. Similar analysis for CDI data was not included due to lack of validated cutoff scores for categorizing symptom severity. Effect of disclosure status on CDI and BDI scores over major assessment time points was evaluated separately using repeated measures analysis with disclosure status entered as time varying covariate [32]. Baseline CDI/BDI score, time, and interaction between time and disclosure status were also included as fixed effects for both models. Two more covariates, baseline HIV Stigma Scale score and Brief IPQ score, were included in the model for BDI score. $P$ value less than 0.05 was considered statistically significant. All analyses were conducted using SAS/STAT ${ }^{\circ}$ software, Version 9.4 of the SAS System for Windows (Cary, NC, USA).

\section{Results}

\section{Characteristics of study participants}

Of the 240 caregiver-child dyads who received the Sankofa intervention at KATH during the parent study, $65 \%(N=$ 157) of them were successfully enrolled in the current study. Of the remaining 83 caregiver-child dyads, 51 dyads were nonresponses-they did not attend clinic during the study period and/or did not respond to phone calls to invite participation. The remaining dyads $(N=32)$ were excluded for the following reasons: child passed $(N=18)$; caregiver passed $(N=11)$; both child and caregiver passed $(N=3)$. Table 1 summarizes the children's demographics; $50.3 \%$ were female, and the median age at enrollment was 10 years (IQR $8-12$ ). The majority attended school (93\%), and mother to child transmission was the most common mode of HIV transmission (79.6\%). The disclosed group was significantly older $(p<0.001)$, had been on ART for a longer period of time $(p=0.014)$, and had a longer mean time between enrollment and the current study $(p<$ 0.001 ). No significant differences were noted between children who were or were not enrolled in this study.

The demographics of the caregivers are presented in Table 2. Caregivers' mean age was $42.22 \pm 9.69$ years, and $82.8 \%$ were female. Fifty-six percent of caregivers were married, and most were living with HIV (61.2\%). Caregivers in the disclosed group were significantly older $(p=0.04)$ and differed in their employment statuses $(p=$ 0.020). Compared to caregivers who were enrolled in the current study, those who were not had significant differences in marital status $(p=0.016)$, monthly household income $(p=0.036)$, and baseline HIV-KQ-18 score ( $p=$ 0.018). Compared to the caregivers not enrolled in the current study, the caregivers enrolled lived significantly closer to KATH $(p=0.046)$.

\section{Depression scores at 'Sankofa' baseline and the current study in children living with HIV and their caregivers}

In the current study, participants' depression scores were collected a median of 5.19 years (IQR 4.16-6.07 years) after participants' respective dates of enrollment in the parent study. The CDI scores of the children were $5.19 \pm$ 3.77 (mean \pm SD) and $3.35 \pm 3.50$ (mean \pm SD) at 'Sankofa' baseline and the current study, respectively. There was a significant mean reduction of 1.82 (95\% CI: 1.01-2.63) between baseline and the current study's measurements $(p<$ 0.0001 ). Interestingly, children who were disclosed to had a greater reduction in CDI scores compared to those who were not disclosed to, although, this did not reach statistical significance. After adjusting for time since enrollment, we still found no significant difference in CDI reduction between disclosed and non-disclosed children $(p=0.19)$. CDI scores of the children in the current study were significantly correlated with BDI scores of the caregivers in the current study $(r=0.19, p=0.019)$.

BDI scores at 'Sankofa' baseline and the current study for caregivers were $6.55 \pm 6.02$ and $3.94 \pm 4.49$, respectively. We observed a statistically significant reduction in BDI scores between baseline and the current study, (2.65 (95\% CI: 1.76-3.55), $p<0.0001$ ). However, we did not observe any significant difference in mean reduction between caregivers of disclosed children compared to caregivers of non-disclosed children. When adjusting for time since enrollment for caregivers, we still did not find a significant difference in BDI reduction between caregivers of disclosed and non-disclosed children $(p=0.41)$. BDI scores from baseline and the current study were found to be highly correlated $(r=0.45, p<0.0001)$. When BDI scores were analyzed as a categorical variable, we observed improvement of scores between baseline and the current study: 6 (86\%) caregivers with mild depression changed to 
Table 1 Child demographics

\begin{tabular}{|c|c|c|c|c|}
\hline & \multicolumn{2}{|l|}{ Disclosure Status } & \multirow[b]{2}{*}{ Total $(N=157)$} & \multirow[b]{2}{*}{$p$ Value } \\
\hline & No $(N=35)$ & Yes $(N=122)$ & & \\
\hline \multicolumn{5}{|l|}{ Gender } \\
\hline Female & 019 (54.29\%) & $060(49.18 \%)$ & 079 (50.32\%) & \\
\hline Male & $016(45.71 \%)$ & $062(50.82 \%)$ & $078(49.68 \%)$ & $0.59^{\mathrm{a}}$ \\
\hline \multicolumn{5}{|l|}{ Child Age } \\
\hline Mean $(95 \% \mathrm{CL})$ & $8.88(8.25-9.51)$ & $10.31(9.86-10.75)$ & $9.99(9.61-10.37)$ & $<0.001^{c, * * *}$ \\
\hline Median (IQR) & $9(7-10)$ & $10(8-12)$ & $10(8-12)$ & \\
\hline N (N Missing) & $33(2)$ & $118(4)$ & $151(6)$ & \\
\hline \multicolumn{5}{|l|}{ School } \\
\hline Yes & $030(85.71 \%)$ & $116(95.08 \%)$ & $146(92.99 \%)$ & \\
\hline No & 002 (5.71\%) & $001(0.82 \%)$ & 003 (1.91\%) & \\
\hline Patient refused to answer & $000(0.00 \%)$ & $001(0.82 \%)$ & $001(0.64 \%)$ & \\
\hline Missing & $003(8.57 \%)$ & 004 (3.28\%) & $007(4.46 \%)$ & $0.20^{b}$ \\
\hline \multicolumn{5}{|l|}{ HIV Transmission Mode } \\
\hline MTC & $025(71.43 \%)$ & $100(81.97 \%)$ & $125(79.62 \%)$ & \\
\hline Other & $001(2.86 \%)$ & $003(2.46 \%)$ & 004 (2.55\%) & \\
\hline Missing & $009(25.71 \%)$ & $019(15.57 \%)$ & $028(17.83 \%)$ & $1.00^{b}$ \\
\hline \multicolumn{5}{|l|}{ Duration of HIV (days) } \\
\hline Mean $(95 \% \mathrm{CL})$ & $1503.60(1006.07-2001.13)$ & $1749.07(1545.42-1952.71)$ & $1702.58(1514.83-1890.32)$ & $0.31^{c}$ \\
\hline Median (IQR) & $1264(406-2513)$ & $1928(747-2588)$ & $1898(745-2579)$ & \\
\hline N (N Missing) & $25(10)$ & $107(15)$ & $132(25)$ & \\
\hline \multicolumn{5}{|l|}{ Duration of ART treatment (days) } \\
\hline Mean $(95 \% \mathrm{CL})$ & $804.92(381.21-1228.64)$ & $1355.88(1162.56-1549.19)$ & $1246.53(1068.69-1424.37)$ & $0.014^{c_{1} *}$ \\
\hline Median (IQR) & $350(32-1208)$ & $1389(406-2145)$ & $1281(147-2107)$ & \\
\hline N (N Missing) & $26(9)$ & $105(17)$ & $131(26)$ & \\
\hline \multicolumn{5}{|l|}{ WHO Staging at time of Diagnosis } \\
\hline Stage 1 & $003(8.57 \%)$ & $016(13.11 \%)$ & $019(12.10 \%)$ & \\
\hline Stage 2 & $006(17.14 \%)$ & $044(36.07 \%)$ & $050(31.85 \%)$ & \\
\hline Stage 3 & $014(40.00 \%)$ & $030(24.59 \%)$ & $044(28.03 \%)$ & \\
\hline Stage 4 & $002(5.71 \%)$ & $013(10.66 \%)$ & 015 (9.55\%) & \\
\hline Missing & $010(28.57 \%)$ & $019(15.57 \%)$ & $029(18.47 \%)$ & $0.10^{\mathrm{a}}$ \\
\hline \multicolumn{5}{|l|}{ HIV+ caregiver } \\
\hline Yes & $017(48.57 \%)$ & $079(64.75 \%)$ & $096(61.15 \%)$ & \\
\hline No or Unsure & $017(48.57 \%)$ & $040(32.79 \%)$ & 057 (36.31\%) & \\
\hline Missing & $001(2.86 \%)$ & $003(2.46 \%)$ & $004(2.55 \%)$ & $0.08^{a}$ \\
\hline \multicolumn{5}{|c|}{ Does anyone help you take your medicine? } \\
\hline Yes & $024(68.57 \%)$ & $101(82.79 \%)$ & $125(79.62 \%)$ & \\
\hline No & $006(17.14 \%)$ & $012(9.84 \%)$ & $018(11.46 \%)$ & \\
\hline Missing & $005(14.29 \%)$ & $009(7.38 \%)$ & $014(8.92 \%)$ & $0.21^{\mathrm{a}}$ \\
\hline \multicolumn{5}{|c|}{ Who helps you with your medicine most of the time? } \\
\hline Biological parent (Mother/Father) & $017(48.57 \%)$ & $075(61.48 \%)$ & $092(58.60 \%)$ & \\
\hline Family (Grandparent/Aunt/Uncle) & $006(17.14 \%)$ & $013(10.66 \%)$ & $019(12.10 \%)$ & \\
\hline Other family member & $001(2.86 \%)$ & $010(8.20 \%)$ & $011(7.01 \%)$ & \\
\hline Other & $000(0.00 \%)$ & $002(1.64 \%)$ & $002(1.27 \%)$ & \\
\hline
\end{tabular}


Table 1 Child demographics (Continued)

\begin{tabular}{|c|c|c|c|c|}
\hline & \multicolumn{2}{|l|}{ Disclosure Status } & \multirow[b]{2}{*}{ Total $(N=157)$} & \multirow[b]{2}{*}{$p$ Value } \\
\hline & No $(N=35)$ & Yes $(N=122)$ & & \\
\hline Unknown & $000(0.00 \%)$ & $001(0.82 \%)$ & $001(0.64 \%)$ & \\
\hline Missing & 011 (31.43\%) & $021(17.21 \%)$ & $032(20.38 \%)$ & $0.55^{b}$ \\
\hline \multicolumn{5}{|c|}{ Days since disclosure } \\
\hline Mean $(95 \% \mathrm{CL})$ & $N A(N A-N A)$ & $1540.62(1467.34-1613.90)$ & 1540.62 (1467.34-1613.90) & \\
\hline Median (IQR) & $N A(N A-N A)$ & $1573(1215-1834)$ & $1573(1215-1834)$ & \\
\hline N (N Missing) & $0(35)$ & $121(1)$ & $121(36)$ & \\
\hline \multicolumn{5}{|c|}{ Days since enrollment } \\
\hline Mean (95\% CL) & $1603.26(1461.08-1745.43)$ & 1914.43 (1851.38-1977.48) & 1845.06 (1783.96-1906.15) & $<0.001^{c, * * *}$ \\
\hline Median (IQR) & $1519(1238-1921)$ & $1941(1624-2243)$ & $1893(1519-2215)$ & \\
\hline
\end{tabular}

Legend: ${ }^{*} p<0.05,{ }^{* * *} p<0.001$

${ }^{a}$ Chi-square test, ${ }^{b}$ Fisher's Exact test, ${ }^{\mathrm{c}} \mathrm{T}$ test

Abbreviations: $A R T$ antiretroviral therapy; $C L$ confidence level; IQR interquartile range; MTC mother-to-child

minimal depression; 5 (100\%) caregivers with moderate depression changed to mild or minimal depression, and 2 (100\%) caregivers with severe depression changed to moderate or minimal depression over time. Only 2 (1.5\%) caregivers changed to mild or moderate depression from minimal depression. These changes did not reach statistical significance (Table 3). However, when the categories were combined into minimal versus mild, moderate, and severe depression, the changes reached statistical significance $(p=0.02)$. We repeated this analysis by disclosure status and found borderline significant improvement in the caregivers of disclosed children $(p=0.06)$ but not in the caregivers of undisclosed children $(p=0.16)$.

\section{Longitudinal trends in depression scores in children living} with HIV and their caregivers

Overall, we did not find a statistically significant association between disclosure status and CDI score of children $(p=0.48)$. The adjusted mean CDI score of children who were disclosed to was lower than that of children who were not disclosed to across all major assessment time points except week 144 . Only at week 48 was the mean difference statistically significant (Fig. 1, Table 4).

A separate mixed model was created for BDI scores of caregivers with baseline HIV Stigma Scale score and Brief IPQ score added as covariates. We did not find a statistically significant association between disclosure status of children and BDI score $(p=0.11)$. Compared to caregivers of non-disclosed children, the adjusted mean BDI score of caregivers of disclosed children was lower at all time points during the parent study and the current study; however, this was not statistically significant except at week 48 (Fig. 2, Table 4).

\section{Discussion}

We conducted a cross-sectional study on caregiver-child dyads a median of 5.19 years (IQR 4.16-6.07 years) after their enrollment in the parent 'Sankofa' study at the intervention site in Kumasi, Ghana, which ended in June 30, 2017. Using screening instruments for depression symptoms, we found statistically significant $(p<0.0001)$ reductions in mean scores for both children living with HIV and their caregivers when compared to baseline values. Contrary to commonly held beliefs in resourcelimited settings, there was no increase in depression symptoms amongst caregiver-child dyads who received a clinic-based, culturally sensitive disclosure intervention. Our findings suggest that intervention-assisted disclosure can allay caregivers' fears surrounding the potential for negative psychological effects after disclosure of HIV status to children living with HIV.

We found a significant mean reduction of 1.82 (95\% CI: $1.01-2.63 ; p<0.0001)$ in CDI scores between baseline and the current study's measurements, a median of 5.19 years (IQR 4.16-6.07 years) after enrollment. This finding suggests that a culturally and developmentally sensitive disclosure process did not worsen depression symptoms. In contrast, a recent report on a disclosure intervention in Kenya showed that children in the intervention arm had higher odds of moving from a less to a more severe depression category on the Patient Health Questionnaire-9 at a time when the prevalence of disclosure in the study cohort had increased [33, 34]. Notably, the difference in odds between the control and intervention arms nearly resolved by the study's last measurement [34], suggesting that the risk of depression is present in the months following disclosure then resolves over time. We did not observe this trend in our data. This likely stems from the proactive nature of the 'Sankofa' study. The trained adherence and disclosure specialists who initiated the disclosure process continued counseling children after disclosure, and the study offered 3 years of intensive psychosocial support [14]. In settings with limited access to mental health resources, 
Table 2 Caregiver demographics

\begin{tabular}{|c|c|c|c|c|}
\hline & \multicolumn{2}{|c|}{ Disclosure Status of Child } & \multirow[b]{2}{*}{ Total $(N=157)$} & \multirow[b]{2}{*}{$p$ Value } \\
\hline & No $(N=35)$ & Yes $(N=122)$ & & \\
\hline \multicolumn{5}{|l|}{ Age caregiver } \\
\hline Mean (SD) & $39.29(10.22)$ & $43.07(9.41)$ & $42.22(9.69)$ & $0.042^{c_{1} *}$ \\
\hline \multicolumn{5}{|l|}{ Marital Status } \\
\hline Divorced or separated & $002(5.71 \%)$ & $011(9.02 \%)$ & $013(8.28 \%)$ & \\
\hline Married & 019 (54.29\%) & 069 (56.56\%) & $088(56.05 \%)$ & \\
\hline Single & 007 (20.00\%) & $014(11.48 \%)$ & $021(13.38 \%)$ & \\
\hline Widowed & 007 (20.00\%) & $028(22.95 \%)$ & $035(22.29 \%)$ & $0.58^{a}$ \\
\hline \multicolumn{5}{|l|}{ Gender } \\
\hline Female & $28(80.00 \%)$ & $102(83.61 \%)$ & $130(82.80 \%)$ & \\
\hline Male & $7(20.00 \%)$ & 20 (16.39\%) & 27 (17.20\%) & $0.59^{a}$ \\
\hline \multicolumn{5}{|l|}{ Caregiver HIV status } \\
\hline No or Unsure & $017(48.57 \%)$ & 040 (32.79\%) & $057(36.31 \%)$ & \\
\hline Yes & 017 (48.57\%) & $079(64.75 \%)$ & $096(61.15 \%)$ & \\
\hline Missing & $001(2.86 \%)$ & $003(2.46 \%)$ & 004 (2.55\%) & $0.08^{a}$ \\
\hline \multicolumn{5}{|l|}{ Education } \\
\hline No School & $006(17.14 \%)$ & $016(13.11 \%)$ & $022(14.01 \%)$ & \\
\hline Elementary Education & $023(65.71 \%)$ & $086(70.49 \%)$ & $109(69.43 \%)$ & \\
\hline Secondary \& Post-secondary Education & $006(17.14 \%)$ & $017(13.93 \%)$ & $023(14.65 \%)$ & \\
\hline Missing & $000(0.00 \%)$ & $003(2.46 \%)$ & $003(1.91 \%)$ & $0.75^{\mathrm{a}}$ \\
\hline \multicolumn{5}{|l|}{ Monthly Household Income } \\
\hline$<50 \mathrm{GHS}$ & $04(11.43 \%)$ & 19 (15.57\%) & $23(14.65 \%)$ & \\
\hline 50-300 GHS & $23(65.71 \%)$ & 78 (63.93\%) & $101(64.33 \%)$ & \\
\hline$>300 \mathrm{GHS}$ & $8(22.86 \%)$ & $23(18.85 \%)$ & $31(19.75 \%)$ & \\
\hline Missing & $0(0.00 \%)$ & $2(1.64 \%)$ & $2(1.27 \%)$ & $0.76^{a}$ \\
\hline \multicolumn{5}{|l|}{ Employment Status } \\
\hline Unemployed & $10(28.57 \%)$ & $13(10.66 \%)$ & $23(14.65 \%)$ & \\
\hline Self-employed & $4(11.43 \%)$ & $10(8.20 \%)$ & $14(8.92 \%)$ & \\
\hline Private/Government Sector & $21(60.00 \%)$ & $99(81.15 \%)$ & $120(76.43 \%)$ & $0.020^{a, *}$ \\
\hline \multicolumn{5}{|c|}{ Family members/other people in home (Child) } \\
\hline Mean (SD) & $4.06(2.24)$ & $3.84(3.36)$ & $3.89(3.13)$ & $0.66^{c}$ \\
\hline N (N Missing) & $35(0)$ & $121(1)$ & $156(1)$ & \\
\hline \multicolumn{5}{|c|}{ Family members/other people in home (Adult) } \\
\hline Mean (SD) & $3.63(2.29)$ & $4.46(3.70)$ & $4.27(3.44)$ & $0.11^{\mathrm{c}}$ \\
\hline N (N Missing) & $35(0)$ & $118(4)$ & $153(4)$ & \\
\hline \multicolumn{5}{|l|}{ Number of living children } \\
\hline Mean (SD) & $3.19(1.87)$ & $2.90(1.88)$ & $2.96(1.87)$ & $0.44^{c}$ \\
\hline N (N Missing) & $32(3)$ & $120(2)$ & $152(5)$ & \\
\hline \multicolumn{5}{|l|}{ Distance from Clinic/Hospital } \\
\hline $0-10 \mathrm{~km}$ & $013(37.14 \%)$ & $046(37.70 \%)$ & $059(37.58 \%)$ & \\
\hline $10-20$ km & $015(42.86 \%)$ & $044(36.07 \%)$ & 059 (37.58\%) & \\
\hline $20-30 \mathrm{~km}$ & $004(11.43 \%)$ & $016(13.11 \%)$ & $020(12.74 \%)$ & \\
\hline More than $30 \mathrm{~km}$ & $003(8.57 \%)$ & $014(11.48 \%)$ & $017(10.83 \%)$ & \\
\hline Missing & 000 (0.00\%) & 002 (1.64\%) & 002 (1.27\%) & $0.90^{a}$ \\
\hline
\end{tabular}


Table 2 Caregiver demographics (Continued)

\begin{tabular}{|c|c|c|c|c|}
\hline & \multicolumn{2}{|c|}{ Disclosure Status of Child } & \multirow[b]{2}{*}{ Total $(N=157)$} & \multirow[b]{2}{*}{$p$ Value } \\
\hline & No $(N=35)$ & Yes $(N=122)$ & & \\
\hline \multicolumn{5}{|l|}{ Receiving own care at } \\
\hline Clinic/Hospital and traditional/homeopathic healer & $000(0.00 \%)$ & $001(0.82 \%)$ & $001(0.64 \%)$ & \\
\hline Clinic/Hospital & $035(100.00 \%)$ & $120(098.36 \%)$ & $155(98.73 \%)$ & \\
\hline Missing & $000(0.00 \%)$ & $001(0.82 \%)$ & $001(0.64 \%)$ & $0.59^{b}$ \\
\hline \multicolumn{5}{|l|}{ Frequency of visits to clinic/hospital } \\
\hline Once or never in last 5 years & 011 (31.43\%) & 045 (36.89\%) & $056(35.67 \%)$ & \\
\hline One or more times a year & $024(68.57 \%)$ & $075(61.48 \%)$ & $099(63.06 \%)$ & \\
\hline Other & $000(0.00 \%)$ & $001(0.82 \%)$ & $001(0.64 \%)$ & \\
\hline Missing & $000(0.00 \%)$ & $001(0.82 \%)$ & $001(0.64 \%)$ & $0.70^{b}$ \\
\hline \multicolumn{5}{|l|}{ Social Provisions Questionnaire Overall Score } \\
\hline Mean (SD) & $71.86(5.75)$ & $71.36(5.95)$ & $71.47(5.89)$ & $0.66^{c}$ \\
\hline N (N Missing) & $35(0)$ & $121(1)$ & $156(1)$ & \\
\hline \multicolumn{5}{|l|}{ HIV Knowledge (HIV-KQ-18) } \\
\hline Mean (SD) & $14.54(1.93)$ & $14.70(1.90)$ & $14.67(1.90)$ & $0.66^{c}$ \\
\hline N (N Missing) & $35(0)$ & $118(4)$ & $153(4)$ & \\
\hline \multicolumn{5}{|l|}{ HIV Stigma Score } \\
\hline Mean (SD) & $39.89(6.48)$ & $41.24(4.88)$ & $40.94(5.29)$ & $0.26^{c}$ \\
\hline N (N Missing) & $35(0)$ & $120(2)$ & $155(2)$ & \\
\hline \multicolumn{5}{|l|}{ IIIness Perception Score (Brief IPQ) } \\
\hline Mean (SD) & $31.34(11.25)$ & $32.25(11.51)$ & $32.05(11.42)$ & $0.68^{c}$ \\
\hline N (N Missing) & $35(0)$ & $120(2)$ & $155(2)$ & \\
\hline
\end{tabular}

Legend: 1.0 Ghanaian cedi is equivalent to 0.17 U.S. dollar; ${ }^{*} p<0.05$

${ }^{a}$ Chi-square test, ${ }^{b}$ Fisher's Exact test, ${ }^{\mathrm{c}} \mathrm{T}$ test

Abbreviations: GHS Ghanaian cedi; $S D$ standard deviation

an emphasis on primary prevention is needed, and the Sankofa study reflects this. Only our week 48 (12 months) measurement showed a statistically significant difference between groups, with the disclosed group having a lower mean CDI score. Depression symptoms in children at our intervention site actually improved between enrollment and the current study.

The challenges of caring for children living with HIV and the impact of caregivers' mental health on children make assessment of caregivers' depression symptoms equally important $[35,36]$. In the present study, we observed statistically significant mean reductions $(p<0.0001)$ in BDI scores between baseline and the current study's measurements. At each time point, adjusted mean BDI scores were consistently lower for caregivers of children who had completed the disclosure process. Caregivers of children living with HIV have been found to have a high prevalence of depression symptoms, and our findings suggest disclosure may help to alleviate the burden in this high-risk group [37, 38]. Interestingly, we also found CDI scores in the current study to be significantly correlated with the BDI scores of caregivers $(r=0.19, p=0.019)$. This finding is encouraging because the 'Sankofa' intervention intentionally focused on the experience of both children and their caregivers, with

Table 3 Changes in Beck Depression Inventory categories between baseline and the current study

\begin{tabular}{|c|c|c|c|c|c|c|}
\hline \multirow{2}{*}{$\begin{array}{l}\text { Baseline BDI } \\
\text { Categories }\end{array}$} & \multicolumn{5}{|c|}{ Current Study BDI Categories } & \multirow{2}{*}{$\begin{array}{l}p \\
\text { value }\end{array}$} \\
\hline & Minimal (No.) & Mild (No.) & Moderate (No.) & Severe (No.) & Total (No.) & \\
\hline Minimal (No.) & 140 & 1 & 1 & 0 & 142 & $0.10^{a}$ \\
\hline Mild (No.) & 6 & 0 & 1 & 0 & 7 & \\
\hline Moderate (No.) & 3 & 2 & 0 & 0 & 5 & \\
\hline Severe (No.) & 1 & 0 & 1 & 0 & 2 & \\
\hline Total (No.) & 150 & 3 & 3 & 0 & 156 & \\
\hline
\end{tabular}

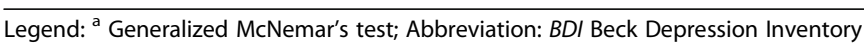




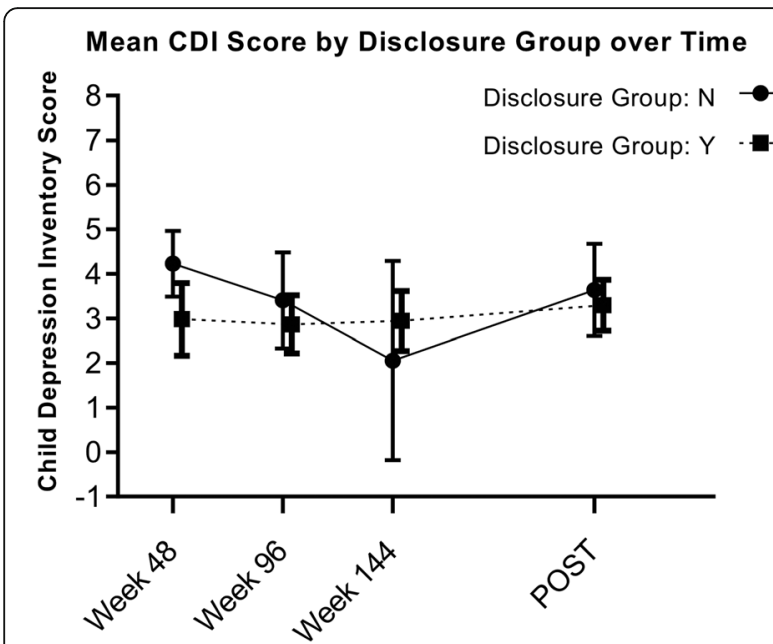

Fig. 1 Depression symptoms in children by disclosure group over time. Disclosure groups represent children who did or did not complete the disclosure process at each time point. Child Depression Inventory (CDI) scores for weeks 48, 96, and 144 were collected during the Sankofa parent study. POST data point was collected during the current study and represents time between enrollment in the parent study and the current study, which varies for study participants (median 5.19 years; IQR 4.16-6.07 years). Effect of disclosure status on CDI scores was evaluated using repeated measures analysis with disclosure status entered as time varying covariate. Abbreviation: CDI, Child Depression Inventory

the disclosure process emphasizing inclusion and mutual empowerment. Caregiver depression symptoms being positively associated with those of children has been previously reported [39], and our observations demonstrate that methods can be successfully developed and deployed to benefit both caregivers and children.

When interpreting our results, there are limitations one needs to consider. First, this exploratory study was only conducted at the intervention site in Kumasi, Ghana. Due to logistical and temporal constraints, we could not parallel the original design of the 'Sankofa' study; however, the non-
Mean BDI Score by Disclosure Group over Time

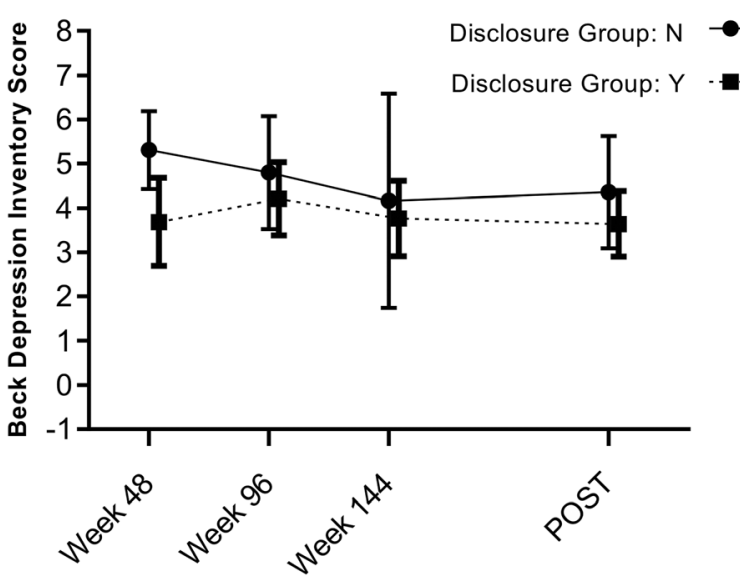

Fig. 2 Depression symptoms in caregivers by disclosure group over time. Disclosure groups represent caregivers of children who did or did not complete the disclosure process at each time point. Beck Depression Inventory (BDI) scores for weeks 48,96 , and 144 were collected during the Sankofa parent study. POST data point was collected during the current study and represents time between enrollment in the parent study and the current study, which varies for study participants (median 5.19 years; IQR 4.16-6.07 years). Effect of disclosure status on BDI scores was evaluated using repeated measures analysis with disclosure status entered as time varying covariate. Abbreviation: BDI, Beck Depression Inventory

disclosed children in the current study serve as controls for the disclosed children because all dyads received the intervention. Our findings show sustained reductions in depression scores for caregiver-child dyads years after the intervention. A second limitation is the use of screening instruments to assess depression symptoms in caregiver-child dyads. They are time-efficient and provide valuable information when analyzed longitudinally, but they are no substitute for a thorough psychiatric evaluation and may be subject to reporting bias. Finally, a number of dyads $(N=$ 83) who received the intervention were not included in this cross-sectional study for various reasons. Compared to

Table 4 Least square means for depression scores over time

\begin{tabular}{|c|c|c|c|c|}
\hline Week & Disclosed LSM (95\% CI) & Non-disclosed LSM (95\% Cl) & Difference of LSM $(95 \% \mathrm{Cl})$ & $p$ value \\
\hline \multicolumn{5}{|c|}{ Least Square Means of BDI } \\
\hline 48 & $5.31(4.43,6.19)$ & $3.69(2.69,4.68)$ & $1.62(0.39,2.85)$ & $0.01^{a}, *$ \\
\hline 96 & $4.8(3.52,6.08)$ & $4.21(3.38,5.04)$ & $0.59(-0.86,2.04)$ & $0.42^{\mathrm{a}}$ \\
\hline 144 & $4.17(1.74,6.59)$ & $3.76(2.91,4.62)$ & $0.4(-2.12,2.93)$ & $0.75^{a}$ \\
\hline post & $4.36(3.09,5.63)$ & $3.64(2.9,4.38)$ & $0.72(-0.69,2.13)$ & $0.32^{\mathrm{a}}$ \\
\hline \multicolumn{5}{|c|}{ Least Square Means of CDI } \\
\hline 48 & $4.23(3.49,4.97)$ & $2.98(2.17,3.8)$ & $1.25(0.2,2.29)$ & $0.02^{a, *}$ \\
\hline 96 & $3.4(2.33,4.48)$ & $2.87(2.22,3.52)$ & $0.54(-0.68,1.76)$ & $0.39^{a}$ \\
\hline 144 & $2.06(-0.18,4.29)$ & $2.95(2.27,3.62)$ & $-0.89(-3.2,1.43)$ & $0.45^{\mathrm{a}}$ \\
\hline post & $3.64(2.61,4.68)$ & $3.3(2.73,3.87)$ & $0.34(-0.81,1.5)$ & $0.56^{\mathrm{a}}$ \\
\hline
\end{tabular}

Legend: * $p<0.05,{ }^{a} \mathrm{~T}$ test; Abbreviations: $B D I$ Beck Depression Inventory; CDI Child Depression Inventory; $C$ confidence interval; LSM least square means 
caregivers who were not enrolled in this current study, enrolled caregivers lived closer to KATH $(p=0.046)$. Most of the enrolled caregivers lived within urban Kumasi, and the heterogeneity of the surrounding areas may not be adequately captured by our study. This study took place over a finite period of time, and limitations on the ability to enroll all original participants does not undermine our findings. Notably, there were no significant differences between the children, and this strengthens our confidence in the results.

\section{Conclusions}

As the prognosis for children living with HIV changes with the expansion of ART, strategies for improving adherence and engagement with care must be developed. Age-appropriate disclosure of HIV status has been recommended by the American Academy of Pediatrics and the WHO $[40,11]$, and our study supports this recommendation's applicability in sub-Saharan Africa. This region is regrettably burdened with $90 \%$ of all children and adolescents living with HIV [1], but it will benefit the most from disclosure interventions which engage children and their caregivers.

\section{Abbreviations}

ART: Antiretroviral therapy; BDI: Beck Depression Inventory; CDI: Child Depression Inventory; IPQ: IIIness Perceptions Questionnaire; KATH: Komfo Anokye Teaching Hospital; WHO: World Health Organization

\section{Acknowledgements \\ We thank the Sankofa Project caregiver and child dyads for their participations. We are grateful to the staff at Pediatric AIDS Clinics at Korle-Bu and Komfo Anokye Teaching Hospitals and the Ghana-Yale Partnership for Global Health for their support. We also wish to acknowledge all members of the Sankofa Study Team. The content of the paper is solely the responsi- bility of the authors and does not necessarily represent the official view of NIH. ClinicalTrials.gov Identifier: NCT01701635.}

\section{Authors' contributions}

CR drafted the manuscript and contributed to study design and data collection. AS assisted with data collection and edited the manuscript. QM assisted with data collection and edited the manuscript. SA provided supervision, contributed to study design, and edited the manuscript. KA assisted with data collection and edited the manuscript. AA assisted with data collection and edited the manuscript. IPO assisted with data collection and edited the manuscript. YX performed statistical analyses and edited the manuscript. YD performed statistical analyses and edited the manuscript. NRR provided supervision, contributed to study design, and edited the manuscript. EP provided supervision, contributed to study design, and assisted in drafting the manuscript. All authors read and approved the manuscript.

\section{Funding}

This study was funded by a grant from National Institutes of Health/National Institute of Child Health and Human Development (R01HD074253). The content of the paper does not necessarily represent the official view of $\mathrm{NIH}$. CR was supported by the Yale University Downs International Health Student Travel Fellowship and the Yale University Lindsay Fellowship for Research in Africa. No funding source was involved in study design, data collection, data analysis, or manuscript drafting. The contents of this paper are solely the responsibility of the authors.

\section{Availability of data and materials}

The datasets used and analyzed during the current study are available from the corresponding author on reasonable request.

\section{Ethics approval and consent to participate}

The study protocol was reviewed and approved by the Institutional Review Boards of Kwame Nkrumah University of Science and Technology/KATH (Protocol \#: CHRPE/AP/146/12) and Yale University (IRB protocol \# 1205010310). All procedures performed in this study were in accordance with the ethical standards of the institutional and/or national research committee and with the 1964 Helsinki declaration and its later amendments or comparable ethical standards. Informed consent was obtained from all individual participants included in the study. Written informed consent was obtained from the caregivers of the children living with HIV, and consent was obtained from caregivers on behalf of children less than 18 years of age with an assent from the child participant.

\section{Consent for publication}

Not applicable.

\section{Competing interests}

The authors declare that they have no competing interests.

\section{Author details}

'Department of Pediatrics, Pharmacology \& Public Health, Yale School of Medicine, 464 Congress Avenue, New Haven, CT 06520, USA. ${ }^{2}$ Department of Child Health, School of Medicine and Dentistry, Kwame Nkrumah University of Science and Technology and Komfo Anokye Teaching Hospital, Kumasi, Ghana. ${ }^{3}$ Yale Center for Analytical Sciences, Yale School of Public Health, New Haven, CT, USA. ${ }^{4}$ Johns Hopkins School of Nursing, Baltimore, MD, USA.

Received: 23 June 2020 Accepted: 12 October 2020

Published online: 20 October 2020

\section{References}

1. Children, HIV, and AIDS: Global snapshot 2019. UNICEF. https:// datauniceforg/resources/children-hiv-aids-global-snapshot/ Published July 2019. Accessed 12 Apr, 2020

2. Davies MA, Gibb D, Turkova A. Survival of HIV-1 vertically infected children. Curr Opin HIV AIDS. 2016 Sep;11(5):455-64.

3. Global HIV \& AIDS statistics - 2019 fact sheet. UNAIDS. https://www.unaids. org/en/resources/ fact-sheet. Accessed 12 Apr, 2020.

4. Bezabhe WM, Chalmers L, Bereznicki LR, Peterson GM. Adherence to antiretroviral therapy and Virologic failure: a meta-analysis. Medicine (Baltimore). 2016 Apr;95(15):e3361.

5. Goga AE, Singh Y, Singh M, Noveve N, Magasana V, Ramraj T, et al. Enhancing HIV treatment access and outcomes amongst HIV infected Children and adolescents in resource limited settings. Matern Child Health J. 2017 Jan;21(1):1-8.

6. Intasan J, Bunupuradah T, Vonthanak S, Kosalaraksa P, Hansudewechakul R, Kanjanavanit $\mathrm{S}$, et al. Comparison of adherence monitoring tools and correlation to virologic failure in a pediatric HIV clinical trial. AIDS Patient Care STDs. 2014 Jun;28(6):296-302.

7. Assemie MA, Alene M, Ketema DB, Mulatu S. Treatment failure and associated factors among first line patients on highly active antiretroviral therapy in Ethiopia: a systematic review and meta-analysis. Glob Health Res Pol. 2019:4(32):019-0120-4 eCollection 2019.

8. Nichols J, Steinmetz A, Paintsil E. Impact of HIV-status disclosure on adherence to antiretroviral therapy among HIV-infected children in resource-limited settings: a systematic review. AIDS Behav. 2017;21(1):59-69.

9. Umeokonkwo CD, Onoka CA, Agu PA, Ossai EN, Balogun MS, Ogbonnaya LU. Retention in care and adherence to HIV and AIDS treatment in Anambra state Nigeria. BMC Infect Dis. 2019;19(1):654 019-4293-8.

10. Beima-Sofie KM, Brandt L, Hamunime N, Shepard M, Uusiku J, John-Stewart GC, et al. Pediatric HIV disclosure intervention improves knowledge and clinical outcomes in HIV-infected Children in Namibia. J Acquir Immune Defic Syndr. 2017;75(1):18-26.

11. World Health Organization. Guideline on HIV disclosure counselling for children up to 12 years of age. Geneva: World Health Organization; 2011. 
12. Doat AR, Negarandeh R, Hasanpour M. Disclosure of HIV Status to Children in Sub-Saharan Africa: A Systematic Review. Medicina (Kaunas). 2019:55(8). https://doi.org/10.3390/medicina55080433.

13. Vreeman RC, Nyandiko WM, Ayaya SO, Walumbe EG, Marrero DG, Inui TS. The perceived impact of disclosure of pediatric HIV status on pediatric antiretroviral therapy adherence, child well-being, and social relationships in a resource-limited setting. AIDS Patient Care STDs. 2010;24(10):639-49.

14. Reynolds NR, Ofori-Atta A, Lartey M, Renner L, Antwi S, Enimil A, et al. SANKOFA: a multisite collaboration on paediatric HIV disclosure in Ghana. AIDS. 2015;29(Suppl 1):S35-45.

15. Kallem S, Renner L, Ghebremichael M, Paintsil E. Prevalence and pattern of disclosure of HIV status in HIV-infected children in Ghana. AIDS Behav. 2011; 15(6):1121-7.

16. Paintsil E, Renner L, Antwi S, Dame J, Enimil A, Ofori-Atta A, et al. HIV knowledge, stigma, and illness beliefs among pediatric caregivers in Ghana who have not disclosed their child's HIV status. AIDS Care. 2015;27(Suppl 1): 18-27.

17. O'Malley G, Beima-Sofie K, Feris L, Shepard-Perry M, Hamunime N, JohnStewart G, et al. "if I take my medicine, I will be strong: " evaluation of a pediatric HIV disclosure intervention in Namibia. J Acquir Immune Defic Syndr. 2015 Jan 1:68(1):e1-7.

18. Vreeman RC, Scanlon ML, Marete I, Mwangi A, Inui TS, McAteer Cl, et al. Characteristics of HIV-infected adolescents enrolled in a disclosure intervention trial in western Kenya. AIDS Care. 2015;27(Suppl 1):6-17.

19. Bronfenbrenner U, Ceci SJ. Nature-nurture reconceptualized in developmental perspective: a bioecological model. Psychol Rev. 1994;101(4):568-86.

20. Fisher JD, Fisher WA, Williams SS, Malloy TE. Empirical tests of an information-motivation-behavioral skills model of AIDS-preventive behavior with gay men and heterosexual university students. Health Psychol. 1994; 13(3):238-50.

21. Fisher JD, Fisher WA, Amico KR, Harman JJ. An information-motivationbehavioral skills model of adherence to antiretroviral therapy. Health Psychol. 2006;25(4):462-73.

22. Fisher JD, Amico KR, Fisher WA, Harman JJ. The information-motivationbehavioral skills model of antiretroviral adherence and its applications. Curr HIV/AIDS Rep. 2008;5(4):193-203.

23. Rivet AK. A situated-information motivation behavioral skills model of care initiation and maintenance (sIMB-CIM): an IMB model based approach to understanding and intervening in engagement in care for chronic medical conditions. J Health Psychol. 2011;16(7):1071-81.

24. Paintsil E, Kyriakides TC, Antwi S, Renner L, Nichols JS, Amissah K, et al. Clinic-based pediatric disclosure intervention trial improves pediatric HIV status disclosure in Ghana. J Acquir Immune Defic Syndr. 2020;84(1):122-31.

25. Beck AT, Steer SR, Brown GK. Beck depression inventory: Texas psychological corporation. Harcourt Brace \& Co: New York; 1996.

26. Broadbent E, Ellis CJ, Thomas J, Gamble G, Petrie KJ. Can an illness perception intervention reduce illness anxiety in spouses of myocardial infarction patients? A randomized controlled trial. J Psychosom Res. 2009; 67(1):11-5.

27. Cutrona CE, Russell DW. The provisions of social relationships and adaptations to stress. Adv Personal Relationships. 1987:1:37-67.

28. Berger BE, Ferrans CE, Lashley FR. Measuring stigma in people with HIV: psychometric assessment of the HIV stigma scale. Res Nurs Health. 2001; 24(6):518-29.

29. Carey MP, Schroder KE. Development and psychometric evaluation of the brief HIV knowledge questionnaire. AIDS Educ Prev. 2002;14:172-82.

30. Kagotho N, Patak-Pietrafesa M, Ssewamala FM, Kirkbride G. Assessing the association between depression and Savings for Kenyan Youth Using a validated child depression inventory measure. J Adolesc Health. 2018;62(1S): S21-8.

31. Kagee A, Nel A, Saal W. Factor structure of the Beck depression inventory-II among south Africans receiving antiretroviral therapy. AIDS Care. 2014 Feb; 26(2):257-62.

32. Fitzmaurice GM, Laird NM, Ware JH. Applied longitudinal analysis. Hoboken, NJ: Wiley; 2004.

33. Kroenke K, Spitzer RL, Williams JB. The PHQ-9: validity of a brief depression severity measure. J Gen Intern Med. 2001;16:606-13.

34. Vreeman RC, Nyandiko WM, Marete I, Mwangi A, McAteer Cl, Keter A, et al. Evaluating a patient-centred intervention to increase disclosure and promote resilience for children living with HIV in Kenya. AIDS. 2019;33(Suppl 1):\$93-\$101.
35. Lentoor AG. Psychosocial challenges associated with caregiving in the context of pediatric HIV in rural eastern cape. Front Public Health. 2017;5: 127.

36. Lentoor AG, Asante KO, Govender K, Petersen I. Psychological functioning among vertically infected HIV-positive children and their primary caregivers. AIDS Care. 2016;28(6):771-7.

37. Lv Y, Zhao Q, Li X, Stanton B, Fang X, Lin X, et al. Depression symptoms among caregivers of children in HIV-affected families in rural China. AIDS Care. 2010 Jun;22(6):669-76.

38. Ofori-Atta A, Reynolds NR, Antwi S, Renner L, Nichols JS, Lartey M, et al. Prevalence and correlates of depression among caregivers of children living with HIV in Ghana: findings from the Sankofa pediatric disclosure study. AIDS Care. 2018 Oct;25:1-10.

39. Kikuchi K, Poudel KC, Rwibasira JM, Majyambere A, Mutabazi V, Nyonsenga SP, et al. Caring for perinatally HIV-infected children: call for mental care for the children and the caregivers. AIDS Care. 2017 Oct;29(10):1280-6.

40. American Academy of Pediatrics Committee on pediatrics AIDS. Disclosure of illness status to children and adolescents with HIV infection. Pediatrics. 1999;103(1):164-6.

\section{Publisher's Note}

Springer Nature remains neutral with regard to jurisdictional claims in published maps and institutional affiliations.

Ready to submit your research? Choose BMC and benefit from

- fast, convenient online submission

- thorough peer review by experienced researchers in your field

- rapid publication on acceptance

- support for research data, including large and complex data types

- gold Open Access which fosters wider collaboration and increased citations

- maximum visibility for your research: over $100 \mathrm{M}$ website views per year

At $\mathrm{BMC}$, research is always in progress.

Learn more biomedcentral.com/submissions 